\title{
Optical transition rates of a meso-substituted thiacarbocyanine in methanol-in-oil reverse micelles
}

\author{
Serdar Özçelik ${ }^{\mathrm{a}, *}$, N. Zeynep Atay ${ }^{\mathrm{b}}$ \\ a Department of Chemistry, Bilkent University, Bilkent-06533, Ankara, Turkey \\ ${ }^{\mathrm{b}}$ Department of Chemistry, Boğaziçi University, Bebek-80815, Istanbul, Turkey
}

Received 8 July 2004; accepted 30 July 2004

\begin{abstract}
We report the photophysical properties of 3,3'-diethyl-5,5'-dichloro-9-phenylthiacarbocyanine (DDPT) in methanolin-oil (m/o) reverse micellar systems which form methanol droplets stabilized with anionic surfactant aerosol-OT (AOT) in n-heptane. The fluorescence quantum yield of DDPT is enhanced by a factor of 17 in the methanol droplet in comparison with bulk methanol. The fluorescence lifetimes of DDPT in m/o reverse micelles are prolonged up to $2.2 \mathrm{~ns}$ with increasing molar ratio of methanol to surfactant $\left(w_{0}=[\mathrm{MeOH}] /[\mathrm{AOT}]\right)$, whereas the fluorescence lifetime of DDPT in bulk methanol is 75 ps. The non-radiative rate constants of DDPT in the droplets are decreased by a factor of 40 , resulting in a remarkable enhancement in quantum yields, indicating that internal motions of DDPT in the droplets are significantly reduced due to strong electrostatic interactions between the positively charged DDPT and the negatively charged sulfonate head-groups of AOT and the spatial confinement induced by the reverse micellar structure.
\end{abstract}

(C) 2004 Elsevier B.V. All rights reserved.

Keywords: Enhanced fluorescence; Cyanine dyes; Reverse micelles; Methanol-in-oil-droplet; Spatial confinement

\section{Introduction}

Utilization of surfactant aggregation in nonaqueous polar solvents, such as ethylene glycol, glycerol, formamide, acetonitrile and methanol,

\footnotetext{
*Corresponding author. Current address: Pacific Northwest National Laboratory, Chemical Sciences Division, 3335 Q Avenue, EMSL, K8-88, Richland, WA 99352, USA. Tel.: + 1509-376-4026; fax: + 1-509-376-6066.

E-mail address: serdar.ozcelik@pnl.gov (S. Özçelik).
}

have been recently of interest [1,2]. Reverse micellar systems consisting of nanometer-sized droplets of non-aqueous polar solvents, dispersed in an oil-continuous phase have potential as novel reaction media. Although, to date, there have been very few reactions studied in these solvent combinations, there are reports on structural studies performed on sodium dodecyl sulphate (SDS)/formamide/hexanol/alkane [3] and pentakis(ethylene glycol)dodecyl ether/glycerol/alkane systems $[4,5]$. Glycerol and water were shown to 
behave in a very similar manner when dispersed in alkane solvents, when aerosol-OT (AOT, see chemical structure in Fig. 1) or cetyltrimethylammonium bromide (CTAB) is used as surfactants [6,7]. AOT- or CTAB-stabilized glycerol-in-oil microemulsions were reported to exhibit metal ion/ligand complexation reactions [8]. The AOT/ formaldehyde (with added $\mathrm{NaBr}$ )/styrene system has been used for studies of polymerization processes [9].

Solvation dynamics of water and non-aqueous polar solvents in reverse micelles were demonstrated to depend on the molar ratio of solvent to surfactant [2,10-15]. Shirota and Horie reported that the solvation dynamics were retarded by up to
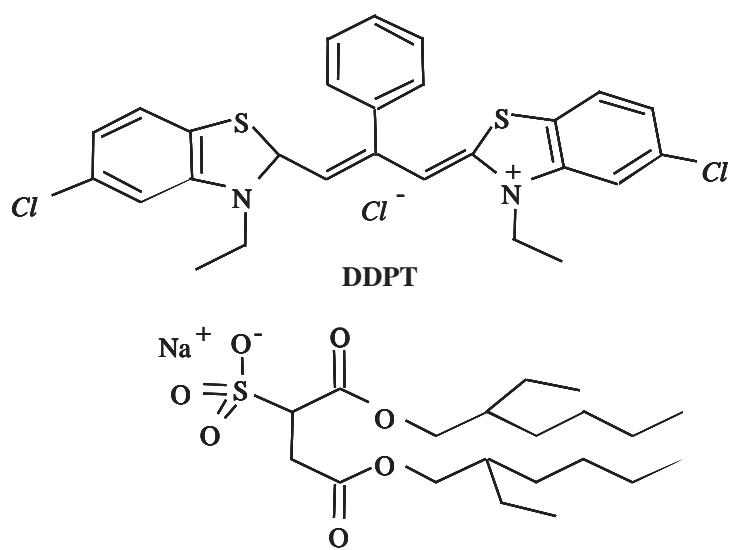

AOT

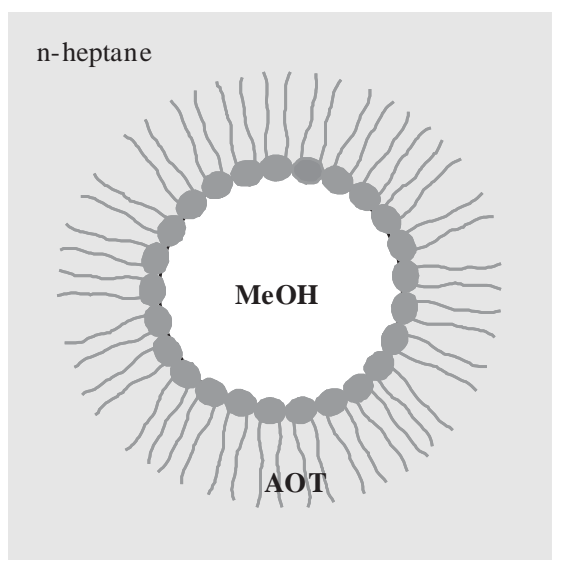

Fig. 1. Chemical structures of DDPT and AOT and a schematic drawing for reverse micelle structure. four orders of magnitude because of the presence of hydrogen-bonding network in reverse micelles [10]. It was proposed that the absence of the hydrogen-bonding network for the non-aqueous solvents such as acetonitrile in reverse micelles causes a weaker interaction between the solvent molecules [10].

Photophysical properties of some cyanine dyes have also been investigated in reverse micelles $[16,17]$. The slower rate of photoisomerisation of a diethyloxacarbocyanine in reverse micelle was proposed to arise from electrostatic interactions between the dye and head group of the surfactant [16]. The position of the dye was thought to be the interface region of the reverse micelles [16]. A meso-substituted cyanine dye structurally similar to DDPT (Fig. 1 for its chemical structure) was employed for the investigation of the isomerization and aggregation in normal and reverse micellar environment [17]. The incorporation of the dye into the micelles resulted in formation of the transisomer, whereas the cis-isomer predominantly existed in bulk water [17]. In reverse micelles the dye forms J-aggregates when water- to- surfactant molar ratio is bigger than five, and only in the presence of an ionic surfactant such as AOT. On the other hand, the optical rate constants of rhodamine dyes in reverse micelles were proved to depend on the refractive index of the surrounding environment [18,19]. Gacoin et al. proposed that reverse micelles could be an accurate system for measuring quantum efficiencies $[18,19]$.

The methanol-in-oil $(\mathrm{m} / \mathrm{o})$ dispersions were prepared using $w_{0}=2,4$ and 6 , where $w_{0}$ is the molar ratio of dispersed methanol to surfactant $\left(w_{0}=[\mathrm{MeOH}] /[\mathrm{AOT}]\right)[10,12-13]$. In the case of water-in oil microemulsions, $w_{0}$ values can be incorporated into an empirical formula to determine the radii of dispersed water droplets in oil [20]. The radius is known to increase with increasing $w_{0}$ values. There is, however, no empirical formula to support the systematic increase of droplet sizes in the case of methanol dispersions. The radii of the AOT-stabilized methanol droplets in n-heptane for $w_{0}=2,4$ and 6 were measured by dynamic light-scattering methods and reported to be $1.3,1.2$, and $1.5 \mathrm{~nm}$, 
respectively [10]. This demonstrates a slight increase in the droplet size as $w_{0}$ value increases.

The core of a dispersed droplet (the methanol pool) and the interfacial region, which is highly charged due to the anionic head-groups of the AOT molecules, and the counter ions in the Stern layer are shown in Fig. 1c. Therefore, it is expected that the positively charged DDPT should be located at the interfacial region due to presence of anionic head-groups of AOT.

The investigation reported in this paper was to characterize the photophysical properties of DDPT in methanol-in-oil $(\mathrm{m} / \mathrm{o})$ reverse micelles as a reference system, and to understand better spectroscopy and dynamics of cyanines in reverse micelles. The focus was on DDPT because it has recently been demonstrated that the fluorescence of DDPT within the J-aggregates in homogeneous aqueous solutions (bulk) was enhanced by 62 times [21]. The nature of the enhancement is due to the aggregation of DDPT responding collectively to the external optical field [21-23].

The photophysical characterization included absorption, steady-state fluorescence spectroscopy, and fluorescence anisotropy and fluorescence lifetime measurements of DDPT in methanol droplets dispersed in n-heptane in the presence of doubly chained anionic surfactant AOT, and in bulk solvents such as methanol $(\mathrm{MeOH})$, ethylene glycol (EG) and glycerol (GLY). Relative fluorescence efficiencies of DDPT in reverse micelles increased by a factor of 17 in comparison with bulk methanol. The fluorescence lifetimes in $\mathrm{m} / \mathrm{o}$ reverse micellar systems increased with increasing $w_{0}$ values. The non-radiative optical transition rates of DDPT were significantly smaller in the dispersed droplets compared with bulk methanol indicating that the degrees of freedom of DDPT were reduced due to spatial and electrostatic confinement within methanol droplets.

\section{Experimental}

Aerosol-OT, bis(2-ethylhexyl) sodium sulfosuccinate, was purchased from Sigma-Aldrich and used without further purification. Methanol, glycerol and ethylene glycol (all spectrophotometric grade) and n-heptane ( $>99 \%$ pure) were obtained from Merck. DDPT was purchased from the Japanese Research Institute for Photosensitizing Dyes, Ltd. (Okayama, Japan) and used as received.

DDPT was dissolved in pure methanol to prepare a $1.25 \mathrm{mM}$ stock solution. An appropriate aliquot of DDPT-containing solution was added to an AOT solution in n-heptane. The resulting dispersion was a clear mixture. The $w_{0}$ values of the dispersions were varied between 2 and 6 . In all cases, the AOT concentration was kept constant at $0.1 \mathrm{M}$. Solutions prepared with $w_{0}=8$ were found to be unstable, and phase separated immediately after mixing.

All measurements were conducted at room temperature. Absorption spectra were recorded using a Cary 5E UV-Vis/NIR spectrophotometer. Steady-state fluorescence spectra were acquired using a SPEX Fluorolog- $\tau 2$ spectrofluorimeter. The fluorescence quantum yields were determined relative to rhodamine $6 \mathrm{G}$ in ethanol $(\Phi=0.95)$ as the reference. Absorbance values, less than 0.1 with $1 \mathrm{~cm}$ cell, for the reference and sample were matched. Right-angle geometry was used. The corrections required for wavelength response of the emission monochromator-photomultiplier combination were made with correction factors supplied by the vendor (SPEX). The slit widths for the excitation and emission monochromators were set to $0.5 \mathrm{~mm}$ (an optical resolution of $2 \mathrm{~nm}$ ).

Steady-state fluorescence anisotropy measurements were carried out by using a spectrometer system consisting of a 1-meter Jobin-Yvon U1000 double monochromator equipped with GaAs photomultiplier and photon-counting electronics. An argon-ion laser at $514.5 \mathrm{~nm}$ with a power of $25 \mathrm{~mW}$ on the sample was the excitation source. A Glann-Thompson prism was used to rotate the state of polarisation of the laser. A polariser in front of the input slit of the Jobin-Yvon U1000 spectrometer was used to analyse polarisation of the emission from the sample.

Fluorescence lifetime measurements were conducted by a Hamamatsu fluorescence lifetime measurement system consisting of a streak camera (Model C4334) optically coupled 
charge-coupled-device (CCD) array detector and a Chromex 250i imaging spectrometer. The ultimate time-resolution using Hamamatsu U4290 fluorescence analysis software was estimated to be slightly better than $10 \mathrm{ps}$ [21-23]. The excitation light source for the lifetime measurements was a Spectra-Physics all solid-state picosecond laser system: optical parametric amplifier (OPA) pumped by Ti:S Spitfire laser that is seeded by a Tsunami laser. The samples were excited at 520 and $575 \mathrm{~nm}$ with pulses of $1.5 \mathrm{ps}$ duration at the repetition rate of $1 \mathrm{kHz}$. The intensity of the incident radiation was adjusted through the use of calibrated neutral density filters, ensuring that maximum of $10^{10}$ photons $/ \mathrm{cm}^{2}$ reached to the samples. Fluorescence from the samples was collected at right-angle and/or front-surface geometry using a $0.3 \mathrm{~mm}$ single optical fiber.

\section{Results and discussion}

Fig. 2 shows the absorption spectra of DDPT in bulk methanol and in the $\mathrm{m} / \mathrm{o}$ reverse micelles stabilized by AOT. The absorption spectrum of DDPT in neat methanol exhibits a band at $566 \mathrm{~nm}$ and a shoulder at $528 \mathrm{~nm}$, which are attributed to the $0 \leftarrow 0$ and $1 \leftarrow 0$ vibronic transitions, respectively. The maximum of absorption band of DDPT in the methanol droplets is shifted to $573 \mathrm{~nm}$. The shift of about $210 \mathrm{~cm}^{-1}$ suggests that the dye molecules are now located in a more polar environment. The band shape and maxima of the absorption spectra remain unchanged regardless of the size of the dispersed droplets. However, the successive raise of the absorbance with increasing $w_{0}$ is due to the higher concentration of DDPT in the reverse micelles.

Fluorescence spectrum of DDPT in bulk methanol shows a band maximum at $578 \mathrm{~nm}$ (see Fig. 3). The band maxima of the fluorescence spectra of DDPT in the reverse micelles are shifted to lower energy and located at $585 \mathrm{~nm}$ for all $w_{0}$ values. The amount of $210 \mathrm{~cm}^{-1}$ shift in fluorescence does not change with $w_{0}$ values. The immediate micro-environment around DDPT is not affected by the molar ratio of the dispersed methanol to AOT. Invariant Stokes shift manifests

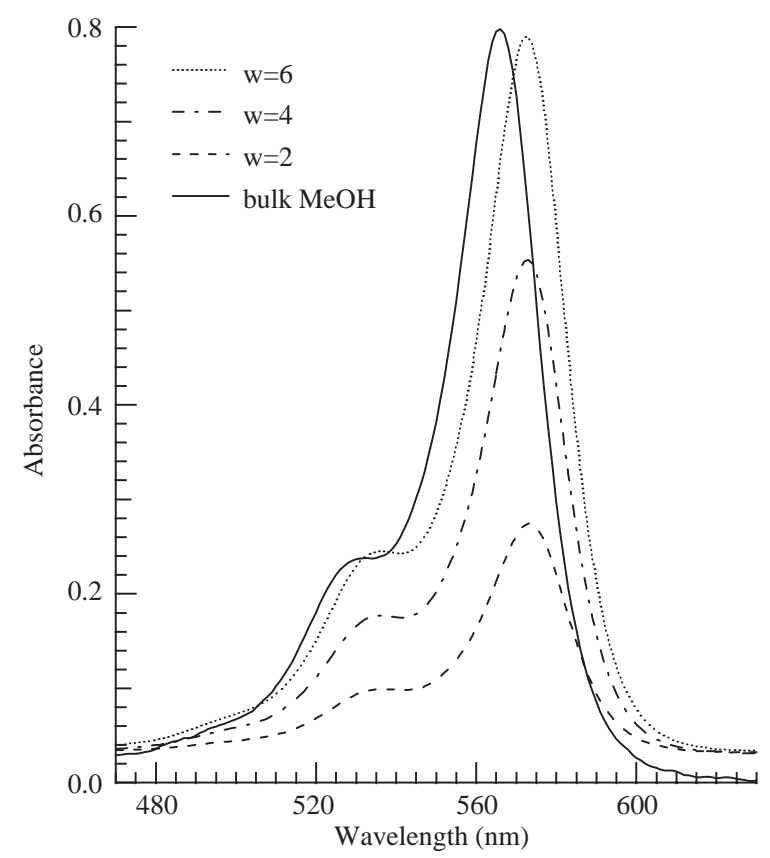

Fig. 2. Absorption spectra of DDPT in $\mathrm{m} / \mathrm{o}$ reverse micelles and bulk methanol. The inserted annotation provides information for the molar ratio of methanol to AOT. The absorbance values for bulk methanol and $w_{0}=6$ solutions are matched for comparison. $[\mathrm{AOT}]=0.1 \mathrm{M} ; w_{0}=2,4$ and 6 . [DDPT] $=$ $1.25 \mathrm{mM}$

that the ground and fluorescent state of DDPT in the droplets should be very similar.

The findings, invariance band-shape and -maxima of the absorption and fluorescence spectra with the size of methanol droplets, point out that the micro-environment around DDPT remains same as the droplet size increases. DDPT has no solubility in the n-alkane phase of $\mathrm{m} / \mathrm{o}$ reverse micellar systems. It is therefore reasonable to assume that DDPT in methanol is incorporated within the methanol droplet. Considering the cationic nature of DDPT, it is very likely that DDPT could be attracted to the anionic sulfonate head-groups of AOT [16]. This interaction should introduce electrostatic confinement in addition to spatial confinement provided by the reverse micellar structure. As a result of the electrostatic interactions, higher activation energy for the solvation processes would be expected [2]. The 


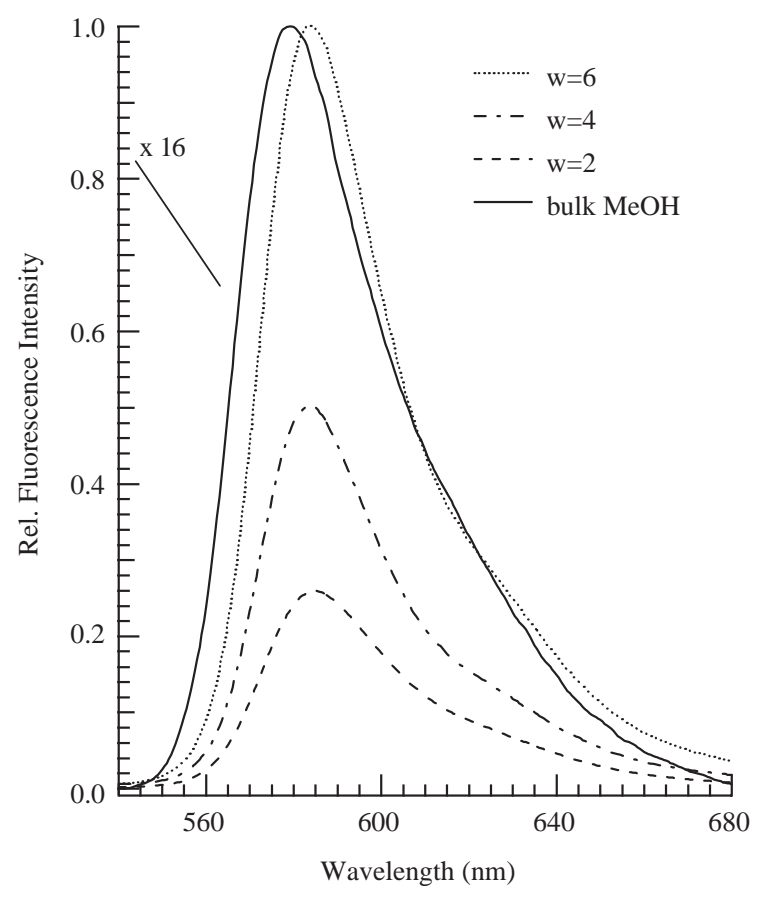

Fig. 3. Fluorescence spectra of DDPT in $\mathrm{m} / \mathrm{o}$ reverse micelles and bulk methanol. Scale factor as tagged to the band for bulk methanol. $[\mathrm{AOT}]=0.1 \mathrm{M} ; w_{0}=2,4$ and $6 .[\mathrm{DDPT}]=1.25 \mathrm{mM}$.

internal motions, for example rotations and vibrations, would be restricted due to electrostatic and spatial confinements leading to slower transitions between the excited states and the ground state.

The photodynamics of DDPT in reverse micelles was investigated to shed light on the photophysical processes. The fluorescence quantum yields of DDPT in the reverse micelles are increased by a factor of 17 compared with that of bulk methanol. This enhancement should arise from the retardation of the non-radiative processes which include internal conversions, intersystem crossings and photoisomerisation. Intersystem crossing, triplet formation and related deactivation channels of the first excited singlet state, i.e. the fluorescent state of cyanines are not very effective and have very low quantum yields [24-32]. Photoisomerisation and internal conversions leading to picosecond fluorescence lifetimes are considered as the main non-radiative deactivation pathways for cyanines
[31,32]. Krieg et al. investigated effects of structural modifications on photophysical properties of thiacarbocyanine dyes in neat solvents and liposomes [33,34]. They concluded that the mesosubstitution of thiacarbocyanine dyes significantly reduces photoisomerisation rate; however, it drastically enhances internal conversions which become the main non-radiative deactivation pathway of the first excited singlet state. Therefore, the internal conversions may be considered as the main non-radiative process.

Fluorescence decay curves of DDPT in m/o reverse micelles and in bulk methanol are depicted in Fig. 4. The fluorescence lifetimes in the methanol droplets vary between 1.5 and $2.2 \mathrm{~ns}$ as a function of $w_{0}$. However, the lifetime in bulk methanol is $75 \mathrm{ps}$. The fluorescence lifetimes in

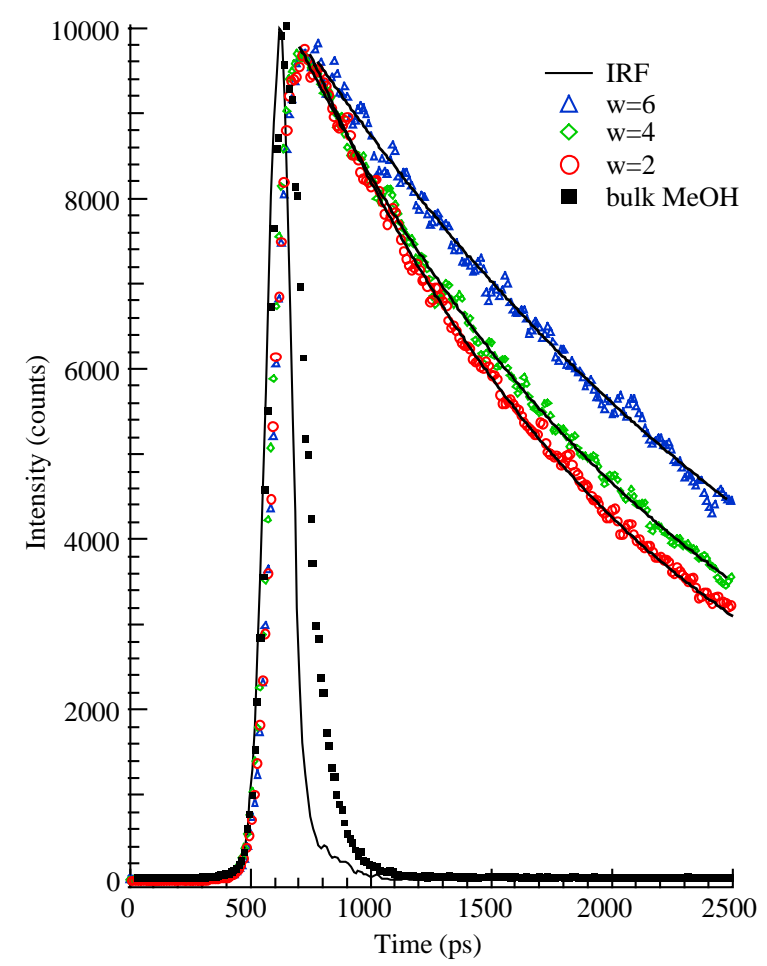

Fig. 4. Fluorescence decay curves of DDPT in $\mathrm{m} / \mathrm{o}$ reverse micelles and bulk methanol. The inserted annotation provides information for the molar ratio of methanol to AOT. The solid line represents the instrument response function (IRF) of the lifetime measurement set-up. The decay curves of the dye in reverse micelles are fitted to a single exponential function. The results are tabulated in Table 1. 
Table 1

Photophysical parameters of DDPT in $\mathrm{m} / \mathrm{o}$ reverse micelles and bulk solvents

\begin{tabular}{llllllrr}
\hline$w_{0}$ & $\tau_{\text {fluo }}(\mathrm{ns})$ & $\phi_{\text {fluo }}$ & $k_{\mathrm{r}} \times 10^{9}\left(\mathrm{~s}^{-1}\right)$ & $k_{\mathrm{nr}} \times 10^{9}\left(\mathrm{~s}^{-1}\right)$ & $k_{\mathrm{r}}^{\text {bulk }} / k_{\mathrm{r}}^{\mathrm{rv}}$ & $k_{\mathrm{nr}}^{\text {bulk }} / k_{\mathrm{nr}}^{\mathrm{rv}}$ & $k_{\mathrm{nr}} / k_{\mathrm{r}}$ \\
\hline 2 & $1.52(1.3)$ & 0.25 & 0.17 & 0.49 & 1.2 & 27 & 3 \\
4 & $1.68(1.3)$ & 0.25 & 0.15 & 0.45 & 1.4 & 29 & 3 \\
6 & $2.21(1.2)$ & 0.25 & 0.11 & 0.34 & 1.8 & 39 & 0.10 \\
MeOH & $0.075(0.8)$ & 0.015 & 0.20 & 13.10 & - & - & 0.11 \\
EG & $0.55(1.2)$ & 0.066 & 0.12 & 1.70 & - & - & 0.06 \\
GLY & $2.04(0.8)$ & 0.105 & 0.05 & 0.45 & - & 9 \\
\hline
\end{tabular}

The values in parentheses in the lifetime column are the $\chi^{2}$ values. The error in the rate constants are less than $2 \%$. The error in the measurement of quantum yields is smaller that $0.1 \%$.

$r$ : anisotropy. rv: reverse micelle.

ethylene glycol (EG) and glycerol (GLY) are also measured, changing from 0.55 to $2.0 \mathrm{~ns}$ (Table 1). The lifetimes are single exponential both in the reverse micelles and in the bulk solvents.

Fluorescence lifetime and quantum yield are related to the radiative $\left(k_{\mathrm{r}}\right)$ and non-radiative $\left(k_{\mathrm{nr}}\right)$ rate constants by the photophysical equations:

$k_{\mathrm{r}}=\Phi_{\mathrm{f}} / \tau_{\mathrm{f}}$,

$k_{\mathrm{nr}}=1 / \tau_{\mathrm{f}}-k_{\mathrm{r}}$.

where $\tau_{\mathrm{f}}$ and $\phi_{\mathrm{f}}$ are, respectively, fluorescence lifetime and quantum yield. The radiative and non-radiative rate constants are tabulated in Table 1. The rate constants of DDPT in the dispersed methanol are smaller compared with the bulk methanol and decrease with increase in the molar ratio of methanol to AOT. The change in the magnitude of the non-radiative rate constant is much greater compared with the radiative rate constants (Table 1). The substantial reduction in the non-radiative rate constant can be understood when the nature of transition is considered. Internal conversion was determined as the origin of non-radiative transitions for meso-substituted thiacarbocyanines [33,34]. Molecular rigidity, steric hindrance and bulk solvent properties such as viscosity and polarity can control the rate of internal conversion. Since steric hindrance leading to photoisomerisation is inhibited upon mesosubstitution, the solvent effects should determine the rate of non-radiative transitions considering the molecular rigidity that is ensured by the spatial confinement provided by the reverse micelle structure. Shirota and Horie measured the solvation dynamics of methanol in reverse micelle and reported that the average solvation times were significantly reduced to nanoseconds in comparison to bulk methanol [10]. The slower solvation dynamics of methanol in reverse micelles is explained by the formation of hydrogen-bonding network between methanol molecules in restricted environment $[2,10]$. Methanol molecules which are oriented by the negatively charged head-groups strongly interact with other molecules located in the core of the reverse micelle $[2,10]$.

Our calculations show that the ratio of radiative to non-radiative rates $\left(k_{\mathrm{nr}} / k_{\mathrm{r}}\right)$ remains constant with increasing $w_{0}$, the molar ratio of methanol to AOT. To understand better the contribution of solvent effects to photodynamics of DDPT in reverse micelles, the rate constants of DDPT in bulk solvents were calculated. The radiative rate constants in bulk EG and AOT-stabilized $\mathrm{m} / \mathrm{o}$ reverse micelles have similar magnitudes. On the other hand, the non-radiative rate constants in bulk GLY and the reverse micelles are comparable. The ratio $k_{\mathrm{nr}} / k_{\mathrm{r}}$ (Table 1 ) in bulk solvents is changed from 65 to 9 with increased solvent viscosity. The reduction in the ratio, which is mainly due to a decrease in the non-radiative rate constant, indicates the role of viscosity. It is worth noting that the similar magnitude of the nonradiative rate constants of DDPT in bulk glycerol and in reverse micelles. It points out that the nonradiative processes could be similar in both environments. Furthermore, the radiative rate constants in the reverse micelles are 2-3 times 
higher compared to the bulk glycerol. Therefore, viscosity alone does not explain the observed enhancement in the fluorescence intensity. Additionally, the radiative rate constant should be independent of viscosity. The ratio $k_{\mathrm{nr}} / k_{\mathrm{r}}$ is further lowered three-fold in the reverse micelles compared to bulk glycerol, resulting in an overall reduction by a factor of twenty-two. As a result, the nature of the additional reduction in the ratio is due to stronger electrostatic interaction arising from the positively charged DDPT and the negatively charged sulfonate in the interface region, and the spatial confinement of the droplet that is reported to be less than $2 \mathrm{~nm}$ [10]. The electrostatic interactions are supposed to put more restrictions on DDPT motions in the reverse micelles.

To estimate the location of DDPT in the reverse micelles, we measured steady-state fluorescence anisotropy of DDPT as a function of $w_{0}$ [35]. Table 1 summarizes steady-state fluorescence anisotropy values, $r$, in the bulk methanol and in the reverse micelles. The anisotropy value was raised to 0.10 in the reverse micelles compared to 0.06 in the bulk; and remains invariable with $w_{0}$. Therefore, DDPT should be partitioned in the interface region of reverse micelles because the orientational polarizability of methanol in the interface region could be higher due to the presence of ionic species in the Stern layer compared to the methanol pool in the core of reverse micelles. The constant anisotropy value is consistent with the spectroscopic findings: no shift in the spectral maxima with $w_{0}$ values. However, an increase in the anisotropy in reverse micelles does not imply any orientation of DDPT or the reverse micelles which freely floats in the solution.

\section{Conclusion}

The photophysical properties of DDPT in methanol-in-oil $(\mathrm{m} / \mathrm{o})$ reverse micellar systems at room temperature were characterized. The fluorescence lifetimes and the quantum yields are increased in reverse micelles compared to bulk methanol. It is demonstrated that the non-radiative rate constant is controlled by the molar ratio of methanol to AOT. The ratio $\left(k_{\mathrm{nr}} / k_{\mathrm{r}}\right)$ is substantially reduced as a result of the microenvironment in reverse micelles. It is concluded that the molecular motion of DDPT in the reverse micelles is more restricted as a result of the electrostatic and spatial confinement introduced by the reverse micellar structure. The electrostatic confinement arises from the interactions between the positively charged DDPT and the negatively charged AOT head-groups at the interfacial region of the dispersed droplets. The spatial confinement originates from nanometer size of the reverse micellar structure. These results clearly demonstrate that the reverse micelles could be a nanoscale supramolecular host to prepare light emitting materials with controlled optical rates. Recently, Sapp and Elliott demonstrated the utilization of polymer encapsulated reverse micelles as solid-state material for incorporating photosensitive dyes [36]. Ability to control spontaneous emission is important because of potential technological applications such as light emitting diodes with high brightness and stability and lasers with lower thresholds to initiate lasing.

Photophysical characterization of DDPT in non-aqueous (such as ethylene glycol and glycerol) and in aqueous reverse micellar systems, and the utilization of reverse micellar system for J-aggregate formation are underway.

\section{Acknowledgments}

We gratefully thank Daniel L. Akins and Atilla Aydinlı for their support. This work is partially supported by a grant TBAG-1939 obtained from Türkiye Bilimsel ve Teknik Araştırma Kurumu (Scientific and Technical Research Council of Turkey).

\section{References}

[1] T. Warnheim, Curr. Opin. Colloid Interface Sci. 2 (1997) 472.

[2] K. Bhattacharyya, Acct. Chem. Res. 36 (2003) 95.

[3] S.E. Friberg, G. Rong, Langmuir 4 (1988) 796.

[4] A. Martino, E.W. Kaler, J. Phys. Chem. 94 (1990) 1627.

[5] A. Martino, E.W. Kaler, Langmuir 11 (1995) 779. 
[6] P.D.I. Fletcher, M. Galal, B.H. Robinson, J. Chem. Soc. Faraday Trans. 80 (1) (1984) 3307.

[7] P.D.I. Fletcher, M. Galal, B.H. Robinson, J. Chem. Soc. Faraday Trans. 81 (1) (1985) 2053.

[8] N.Z. Atay, B.H. Robinson, Langmuir 15 (1999) 5056.

[9] K.V. Schubert, K.M. Lusvardi, E.W. Kaler, Colloid Polym. Sci. 274 (1996) 875.

[10] H. Shirota, K. Horie, J. Phys. Chem. B. 103 (1999) 1437.

[11] A. Datta, D. Mandal, S.K. Pal, K. Bhattacharyya, J. Phys. Chem. B 101 (1997) 10221.

[12] P. Hazra, D. Chakrabarty, N. Sarkar, Chem. Phys. Lett. 371 (2003) 553.

[13] P. Hazra, D. Chakrabarty, N. Sarkar, Langmuir 18 (2002) 7872.

[14] B. Bangar Raju, S.B.M. Costa, J. Phys. Chem. B 103 (1999) 4309.

[15] R.E. Riter, J.R. Kimmel, E.P. Undiks, N.E. Levinger, J. Phys. Chem. B 101 (1997) 8292.

[16] A. Datta, D. Mandal, S.K. Pal, K. Bhattacharyya, Chem. Phys. Lett. 278 (1997) 77.

[17] A.S. Tatikolov, S.M.B. Costa, Chem. Phys. Lett. 346 (2001) 233.

[18] P. Lavallard, M. Rosenbauer, T. Gacoin, Phys. Rev. A 54 (1996) 5450.

[19] G. Lamouche, P. Lavallard, T. Gacoin, Phys. Rev. A 59 (1999) 4668.

[20] J.D. Nicholson, J.H.R. Clarke, in: K. Mittal, B. Lindman (Eds.), Proceedings of the International Symposium on
Surfactants in Solution, Vol. 3, Plenum Press, New York, 1984, p. 1663.

[21] S. Ozcelik, D.L. Akins, J. Phys. Chem. B 103 (1999) 8926.

[22] S. Ozcelik, I. Ozcelik, D.L. Akins, Appl. Phys. Lett. 73 (1998) 1946.

[23] S. Ozcelik, D.L. Akins, Appl. Phys. Lett. 71 (1997) 3057.

[24] A.V. Buettner, J. Chem. Phys. 46 (1967) 1398.

[25] D.F. O’Brien, T.M. Kelly, L.F. Costa, Photogr. Sci. Eng. 18 (1974) 76.

[26] C.J. Tredwell, C.M. Keary, Chem. Phys. 43 (1979) 307.

[27] S. Ozcelik, J. Lumin. 96 (2002) 141.

[28] J. Jaradus, J. Photochem. 13 (1980) 35.

[29] V. Sundstrom, T. Gillbro, J. Chem. Phys. 78 (1982) 1788.

[30] C. Rulliere, Chem. Phys. Lett. 43 (1976) 303.

[31] X. Shi, E. Bourget, A.N. Tarnovsky, K.B. Eisenthal, Chem. Phys. 205 (1996) 167.

[32] D.F. Aramendia, R.M. Negri, E.S. Roman, J. Phys. Chem. 98 (1996) 3165.

[33] M. Krieg, R.W. Redmond, Photochem. Photobiol. 57 (1993) 472.

[34] M. Krieg, J.M. Bilitz, M.B. Srichai, R.W. Redmond, Biochim. Biophys. Acta 1199 (1994) 149.

[35] J.R. Lakowicz, In Principles of Fluorescence Spectroscopy, second ed., Kluwer Academic/Plenum Publishers, New York, 1999, p. 299.

[36] S.A. Sapp, C.M. Elliott, Chem. Mater. 15 (2003) 1237. 